\title{
Damage Assessment of the Rolling Bearing Based on the Rigid-flexible Coupling Multi-body Vibration Model
}

\author{
Zhou Chang (ORCID: 0000-0002-2619-0290)1 ${ }^{1}$, Lai Hu (ORCID: 0000-0002-7715-5612)² \\ ${ }^{1}$ School of mechanical and electrical engineering, Lanzhou Jiaotong University, Lanzhou, Gansu, P.R. China \\ 730070 E-mail: starismyfriend@163.com \\ ${ }^{2}$ State Key Laboratory for Manufacturing System Engineering, Xi'an Jiaotong University, Xi'an, Shaanxi, P.R. \\ China, 710054
}

In the present study, local defects in deep groove ball bearings are studied as forward and inverse problems. To this end, the separation-integration method is applied for modeling the forward problem. It is assumed that the inner race of the rolling element is multi-DOF, while the outer race is deformable along the radial direction. Then the problem is modeled with concepts of the finite element method. The contact force for the rolling elements is described by the nonlinear Hertz contact deformation. Various surface defects originating from local deformations are introduced into the developed model. Since the outer ring can be coupled with the FE model of the housing, the developed bearing model is capable of considering the transmission path of the bearing housing. Then model parameters are modified to reach better performance in predicting local defects. Through translating the inverse problem into the comparison of the geometric distance, measured indicators are used in the defect detection process and the relative location and size of defects are predicted. Finally, the defect range is established to evaluate the fault severity. Obtained results demonstrate that the proposed method is effective and accurate in the studied cases.

Keywords: deep groove ball bearings; quantitative evaluation; the forward and inverse problems; defect range

\section{Introduction}

With the advent of new technologies, high speed, heavily loaded and high precision bearings require long-term, precise and stable operation under extreme working conditions. Recent studies show that multiple parameters have a strong coupling effect on the operating processes, which adversely affect the performance of the rolling bearing. More specifically, these factors may result in different damages such as fatigue spalling, pitting corrosion and severe elastic-plastic deformations in the contact area of the moving pair. These damages increase vibration noise, drastically change the clearance of moving pairs and affect motion accuracy.

In order to manage this issue, a dynamic model has been proposed to reveal the evolution process of the bearing service state. Based on the Hertz contact theory, Sunnersjo described the correlation between load and displacement and established a 2-DOF vibration model of the rolling bearing, and obtained the vibration response of the inner ring, ignoring the mass and contact performance of the rolling element (SUNNERSJO C S, 1978). Moreover, Rafsanjani simulated the local defects and studied the influence of defects on the nonlinear vibration of the bearing (RAFSANJANI A, 2009). Liew proposed a 5-DOF analysis model considering the axial stiffness of the angular contact ball bearing. Sopanen and Mikkola established a 6-DOF model using multibody dynamics MSC ADAMS software. In the proposed model, the influence of the rolling element is ignored (SOPANEN J, 2003). Reviewing the literature indicates that bearings are widely adopted as a multi-body dynamic system with no ring deformation. Accordingly, many investigations have been conducted so far to study the vibration behavior of bearings in different applications. In other words, the majority of researches in this area are based on this assumption, while only a few studies focused on bearings coupled with the transfer path. This may be attributed to the lack of an effective method to combine time-varying parameters of the bearing under actual working conditions with the elastic ring of the bearing pedestal. In this case, nonlinear factors such as the vibration transmission characteristics of the joint can be hardly embedded into the overall vibration equation of the elastic body. Consequently, the prediction response cannot approach reality, thereby limiting the application. Considering these shortcomings, it is intended to apply the embedded integrated modeling method to study the performance of the deep groove ball bearing.

With the rapid development of science and tech- 
nology, a quantitative evaluation is an inevitable process in the field of mechanical diagnosis. More specifically, evaluating the damage degree of the rolling bearing is considered in this article as the research objective. Starting from the forward dynamic problem, the damage model of the bearing surface is established, and the essential symptom of damage reflected by the dimensionless index after feature enhancement is obtained. Then different damage locations and damage intervals are subdivided as variables to form the characteristic matrix. The inverse problem is transformed into the comparison of space distances. The characteristic value of the measured signal is taken as input, the sample point nearest to the input value is solved through an appropriate distance function and then the location and a specific degree of damage are predicted.

\section{Analysis model of the ring bearing housing}

In order to consider the effects of the ring deformation, vibration of the ring bearing housing is modeled as the bending vibration of the beam. Fig. 1 shows the integrated analysis model in this regard.

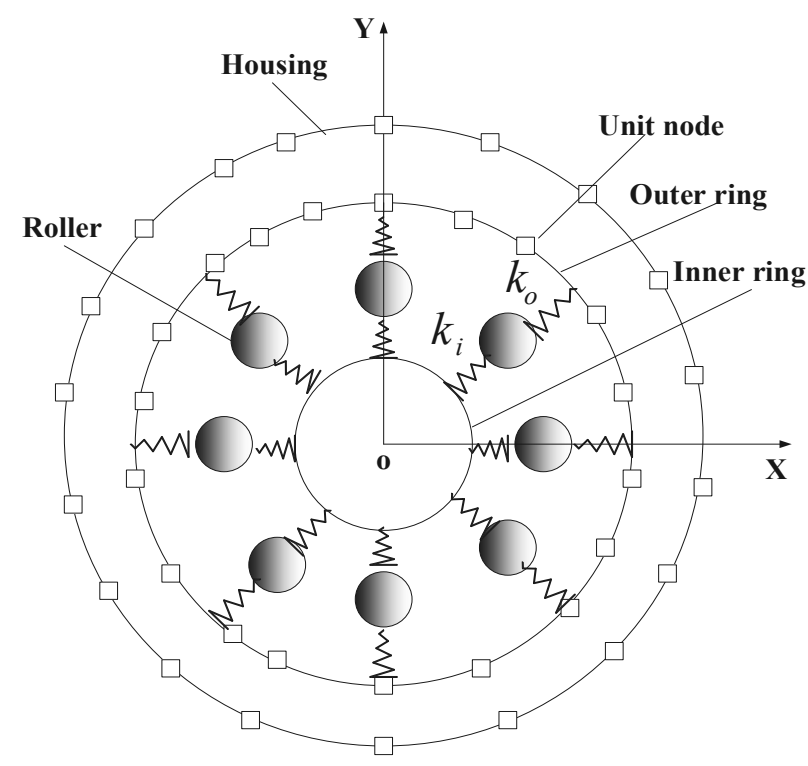

Fig. 1 Developed bearing model

It is worth noting that the actual ring bearing housings are made of elastomers, which intrinsically have a continuously distributed mass and elasticity. Due to the key factors such as the clearance between the rolling element and the inner and outer raceways, flexible multi-body contact and rotational speed, it is an enormous challenge to carry out the finite element calculation of the continuous system directly. Therefore, the finite element theory and multi-body dynamics are applied in the present study to propose an embedded integrated analysis method. The proposed method relies on the idea of "first independent, then embedded". The basic concept of FEA is that the solution domain consists of many interconnected subdomains called finite elements. Then an appropriate approximate solution is assumed for each element and general satisfaction conditions are derived for solving this domain to obtain the final solution of the problem. It should be indicated that FEA not only has a high calculation accuracy but also can be applied in various complex shapes. Differential equation of the system motion can be established by assembling the motion equation of the ring bearing housing as the following:

$$
[\boldsymbol{M}]\{\ddot{\boldsymbol{X}}\}+[\boldsymbol{C}]\{\dot{\boldsymbol{X}}\}+[\boldsymbol{K}]\{\boldsymbol{X}\}=\{\boldsymbol{F}\} .
$$

Where $[\boldsymbol{M}],[\boldsymbol{C}]$ and $[\boldsymbol{K}]$ are the mass matrix, damping matrix and stiffness matrix of the system, respectively. Moreover, $\{\boldsymbol{F}\}$ and $\{\boldsymbol{X}\}$ denote the excitation force and the displacement vector, respectively. The Rayleigh damping matrix can be expressed as a linear combination of mass and stiffness matrices in the form below.

$$
\boldsymbol{C}=\alpha_{\mathrm{R}} \boldsymbol{M}+\beta_{\mathrm{R}} \boldsymbol{K} .
$$

Since the position of the contact force between the rolling element and the outer ring changes continuously along the elastic beam, the exciting force can be expressed as:

$$
\boldsymbol{F}=\sum_{i=1}^{n} \boldsymbol{N}_{i}^{\mathrm{T}} \boldsymbol{F}_{\mathrm{o}, i} .
$$

Where $\boldsymbol{N}_{i}^{\mathrm{T}}, \boldsymbol{F}_{\mathrm{o}, i}$ and $n$ are the displacement interpolation function vector, the contact force of the first rolling element acting on the outer ring and the number of rolling elements, respectively.

\section{Dynamic contact theory of bearing inter- face}

\subsection{Load-displacement relationship}

Based on the Hertz theory, the correlation between load and displacement of the point contact can be mathematically expressed in the form below:

$$
F=K \delta^{1.5} \text {. }
$$

Where $\delta$ is the elastic approach and $K$ denotes the Hertz contact stiffness. In order to describe the contact condition between two meshing surfaces $K_{\mathrm{i}}$ and $K_{0}, \sum \rho$ is defined as the sum of contact stiffness and curvature between the rolling element and the inner and outer rings.

$$
K_{\mathrm{i}}=\frac{2 \sqrt{2}}{3}\left(\frac{E}{1-v^{2}}\right)\left(\sum \rho_{\mathrm{i}}\right)^{-0.5}\left(\mu_{\mathrm{i}}^{*}\right)^{-1.5} .
$$




$$
K_{\mathrm{o}}=\frac{2 \sqrt{2}}{3}\left(\frac{E}{1-v^{2}}\right)\left(\sum \rho_{\mathrm{o}}\right)^{-0.5}\left(\mu_{\mathrm{o}}^{*}\right)^{-1.5} .
$$

Where $E$ is the elastic modulus, $v$ denotes the Poisson's ratio and $\mu^{*}$ is the deformation coefficient. In this way, once the geometric size of the bearing is determined, $K_{\mathrm{i}}$ and $K_{0}$ can be calculated through equations (5) and (6), respectively.

\subsection{Coordinate expression of the interface contact}

As shown in Fig. 2, elastic approach of the first roller ring $\delta_{i}$ is a function of the ring displacement along $\mathrm{X}$ - and Y-directions $\left(x_{\mathrm{s}}, y_{\mathrm{s}}\right)$, position angle $\theta_{i}$ of the rolling body and the clearance $e$. Elastic approach can be calculated through the following equation:

$$
\begin{aligned}
& \delta_{i}=x_{\mathrm{s}} \cos \theta_{i}+y_{\mathrm{s}} \sin \theta_{i}-e . \\
& i=1,2, \cdots, n
\end{aligned}
$$

The position angle of the rolling body can be expressed as $\theta_{i}=\frac{2 \pi}{n} i+\omega_{\mathrm{c}} t$, where $\omega_{\mathrm{c}}$ is the rotating speed of the inner ring and $n$ is the number of steel balls. Moreover, $\omega, D_{\mathrm{b}}$ and $D_{\mathrm{p}}$ denote the shaft speed, diameter of the rolling element and the diameter of the bearing pitch circle, respectively. Accordingly, $\mathrm{xxx}$ speed can be calculated as $\omega_{\mathrm{c}}=\frac{1}{2} \omega\left(1-\frac{D_{\mathrm{b}}}{D_{\mathrm{p}}}\right)$. In an arbitrary point on the mo-

$$
\delta_{i}^{\text {outer }}=\left(x_{\mathrm{b} i}-\boldsymbol{N}_{i}^{\mathrm{e}} \cdot \boldsymbol{X}^{\mathrm{e}}\right) \cos \theta_{i}+\left(y_{\mathrm{b} i}-\boldsymbol{N}_{i}^{\mathrm{e}} \cdot \boldsymbol{Y}^{\mathrm{e}}\right) \sin \theta_{i} .
$$

Where $\boldsymbol{N}_{i}^{\mathrm{e}}$ is the interpolation function vector when the rolling element is in a local contact with the outer ring and $\boldsymbol{X}^{\mathrm{e}}$ denotes the node displacement of the element. In this case, the total elastic recovery force of the outer ring can be expressed as the following:

$$
F_{\mathrm{o} i}=K_{\mathrm{i}}\left[\delta_{i}^{\text {outer }}\right]^{1.5} .
$$

It should be indicated that since the load area has an angle range, $r_{\mathrm{b} i}, \delta_{i}^{\text {inner }}$ and $\delta_{i}^{\text {outer }}$ only can take positive values. The foregoing analysis indicates that the outer ring displacement $\left(x_{0}, y_{0}\right)$ induced by the deformation can be expressed by the average displacement of element nodes. This can be mathematically expressed in the form below: tion path $\left(x_{\mathrm{b}}, y_{\mathrm{b}}\right)$, the corresponding radial displacement can be calculated from the following expression:

$$
r_{\mathrm{b} i}=x_{\mathrm{b} i} \cos \theta_{i}+y_{\mathrm{b} i} \sin \theta_{i} \text {. }
$$

Therefore, the elastic approach of the first $i$ rolling element of the inner ring is:

$$
\delta_{i}^{\text {inner }}=\left(x_{\mathrm{s}}-x_{\mathrm{b} i}\right) \cos \theta_{i}+\left(y_{\mathrm{s}}-y_{\mathrm{b} i}\right) \sin \theta_{i}-e \text {. }
$$

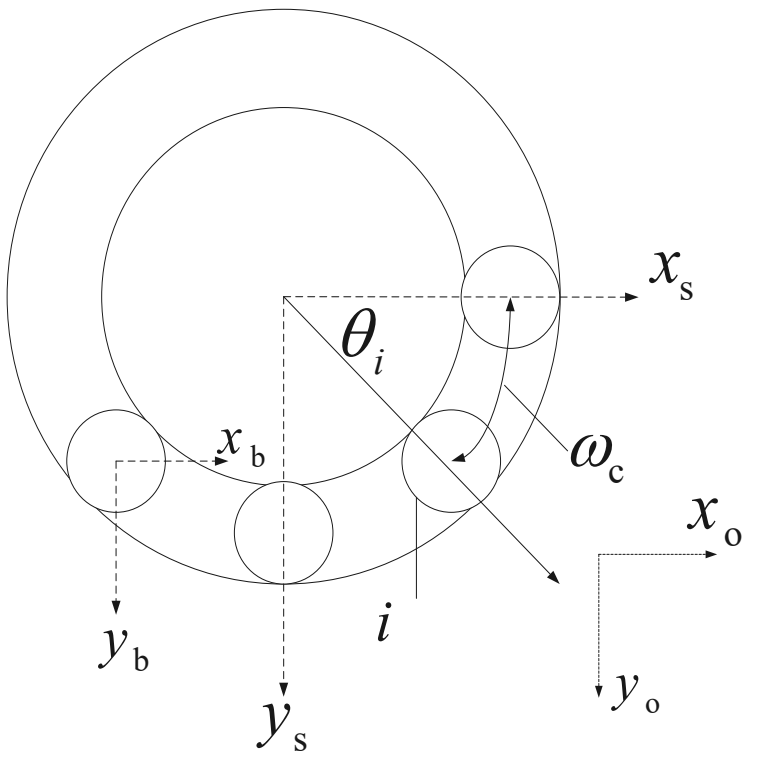

Fig. 2 Schematic diagram of the rolling bearing

In an integrated model, the deformation of the ouequivalent to discretize the whole beam into a combination of finite elements. Accordingly, the elastic approach of the first rolling element of the outer ring can be obtained through the following expression: ter ring and bearing housing are considered. This is

$$
\begin{aligned}
& x_{\mathrm{o}}=\frac{\sum_{j=1}^{N} X_{j}}{N} . \\
& y_{\mathrm{o}}=\frac{\sum_{j=1}^{N} Y_{j}}{N} .
\end{aligned}
$$

\subsection{Equation of motion}

Vibration of the rolling bearing is an excellent embodiment of internal geometric correlations and motion correlations under an external load. Studies show that there is an interaction between vibration source and vibration transmission path. Equations (10) - (13) express the local contact between the deformed ring bearing housing and the rolling element. The finite element model of the ring bearing housing is embedded into the inner ring rolling element transmission path 
composed of mass, stiffness and geometric position. Then the following vibration differential equations can be established based on the Lagrange equation. The integration of equations (1) and (14) shows the internal correlation between the composite constraint and the multi-body contact deformation.

$$
\begin{aligned}
& \int m_{\mathrm{s}} \ddot{\mathrm{x}}_{\mathrm{s}}+\sum_{i=1}^{n} K_{\mathrm{i}}\left[\delta_{i}^{\text {imerer }}\right]^{1.5} \cos \theta_{i}=f_{\mathrm{x}} \\
& m_{\mathrm{s}} \ddot{\boldsymbol{y}}_{\mathrm{s}}+\sum_{i=1}^{n} K_{\mathrm{i}}\left[\delta_{i}^{\text {inner }}\right]^{1.5} \sin \theta_{i}=f_{\mathrm{y}} \\
& m_{\mathrm{b} 1}\left(\ddot{\boldsymbol{x}}_{\mathrm{b} 1}-\ddot{\boldsymbol{x}}_{\mathrm{o}}\right)-\left\{K_{\mathrm{i}}\left[\delta_{1}^{\text {iner }}\right]^{1.5}-K_{\mathrm{o}}\left[\delta_{1}^{\text {outer }}\right]^{1.5}\right\} \cos \theta_{1}=0 \\
& m_{\mathrm{bl}}\left(\ddot{\boldsymbol{y}}_{\mathrm{b} 1}-\ddot{\boldsymbol{y}}_{\mathrm{o}}\right)-\left\{K_{\mathrm{i}}\left[\delta_{1}^{\text {iner }}\right]^{1.5}-K_{\mathrm{o}}\left[\delta_{1}^{\text {outer }}\right]^{1.5}\right\} \sin \theta_{1}-m_{\mathrm{b} 1} g=0 \\
& \left\{m_{\mathrm{b} 2}\left(\ddot{\boldsymbol{x}}_{\mathrm{b} 2}-\ddot{\boldsymbol{x}}_{\mathrm{o}}\right)-\left\{K_{\mathrm{i}}\left[\delta_{2}^{\text {imerer }}\right]^{1.5}-K_{\mathrm{o}}\left[\delta_{2}^{\text {outer }}\right]^{1.5}\right\} \cos \theta_{2}=0\right. \\
& m_{\mathrm{b} 2}\left(\ddot{\boldsymbol{y}}_{\mathrm{b} 2}-\ddot{\boldsymbol{y}}_{\mathrm{o}}\right)-\left\{K_{\mathrm{i}}\left[\delta_{2}^{\text {imer }}\right]^{1.5}-K_{\mathrm{o}}\left[\delta_{2}^{\text {ouler }}\right]^{1.5}\right\} \sin \theta_{2}-m_{\mathrm{b} 2} g=0
\end{aligned}
$$

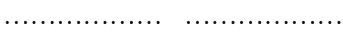

$$
\begin{aligned}
& m_{\mathrm{bn}}\left(\ddot{x}_{\mathrm{b} n}-\ddot{\boldsymbol{x}}_{\mathrm{o}}\right)-\left\{K_{\mathrm{i}}\left[\delta_{n}^{\text {iner }}\right]^{1.5}-K_{\mathrm{o}}\left[\delta_{n}^{\text {outer }}\right]^{1.5}\right\} \cos \theta_{n}=0 \\
& m_{\mathrm{bn}}\left(\ddot{\boldsymbol{y}}_{\mathrm{b} n}-\ddot{\boldsymbol{y}}_{\mathrm{o}}\right)-\left\{K_{\mathrm{i}}\left[\delta_{n}^{\text {imer }}\right]^{1.5}-K_{\mathrm{o}}\left[\delta_{n}^{\text {outer }}\right]^{1.5}\right\} \sin \theta_{n}-m_{\mathrm{bn}} g=0 .
\end{aligned}
$$

Where $m_{\mathrm{s}} 、 m_{\mathrm{b}} 、 m_{0}$ are the mass of the inner ring and shaft, and the mass of the rolling element and the outer ring, respectively. Moreover, $f_{\mathrm{x}} 、 f_{\mathrm{y}} \mathrm{de}-$ notes the sum of radial loads and unbalanced forces. Then boundary conditions are introduced in the same way as that of the finite element method. Rows and columns of the established matrix correspond to the fixed joints of the stiffness matrix. Then the uniform mass matrix is eliminated. The system response can be obtained by solving equation (14) through the Newmark method.

In the process of solving the forward problem, due to the influence of the engineering environment, installation conditions and other factors, the material characteristics, geometric dimensions and boundary conditions of the bearing housing joint may have small deviations. Accordingly, In order to simulate the practical service condition and facilitate the calculation of actual health signal, $[\boldsymbol{C}]$ and $[\boldsymbol{K}]$ are modified through the spectrum of the health signal. The information distance between two signal spectrums can quantitatively represent the different degrees of two spectra. It is worth noting that the smaller the pseudorange of the spectrum, the higher the similarity degree. Considering the symmetry of distance, the J-divergence concept (QU Liang-sheng, 2009). is applied in the present study as the spectrum evaluation algorithm.

$$
J\left(y_{1}, y_{2}\right)=\frac{1}{2 m} \sum_{j=1}^{m}\left(\frac{Q_{1 j}}{Q_{2 j}}+\frac{Q_{2 j}}{Q_{1 j}}-2\right) .
$$

Where $J\left(y_{1}, y_{2}\right)$ is the $\mathrm{J}$-divergence of the spectrum of $y_{1}$ health signal and $y_{2}$ simulation signal, and $m$ is the number of spectral lines. Moreover, $Q_{1 j}$ and $Q_{2 j}$ denote the amplitude of the first $j$ spectral line.

\section{Local damage modeling}

After a long time service, local damages may occur in the bearing, which mainly originates from fatigue. The fatigue site is not a point, but a limited area. Fig. 3 presents the schematic position of the fatigue damage in the bearing. Depth, width and the included angle of the damaged part are $h, 1$ and xxx, respectively. When the rolling element reaches the damaged position, the elastic approach $\delta$ increases the damage depth h instantaneously. Meanwhile, the clearance $e$ in equations (7) and (9) also increase $\mathrm{H}$.

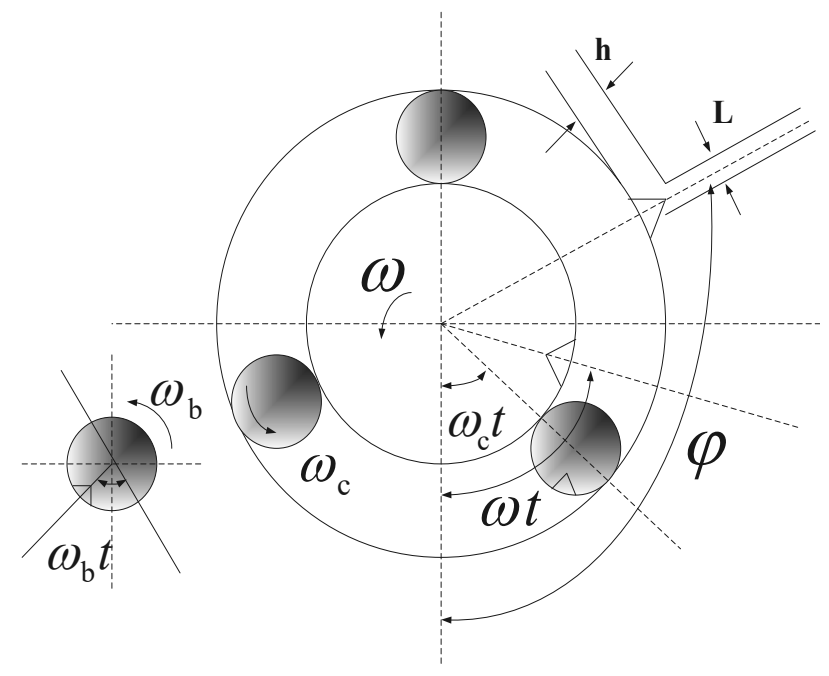

Fig. 3 Schematic position of local defects

It is assumed that $r_{\mathrm{i}}$ and $r_{0}$ are the radius of curvature of the inner and outer rings, respectively. Moreover, $\omega_{\mathrm{b}}$ denotes the rotational speed of the rolling element. When the outer ring is damaged, the damage angle can be determined by the following expression:

$$
\theta_{t}=\theta_{i} \pm \frac{L}{2 r_{0}} \text {. }
$$

On the other hand, when the inner ring is damaged, the damage angle is as follows:

$$
\theta_{t}=\omega t \pm \frac{L}{2 r_{\mathrm{i}}}
$$

Furthermore, in the case when the ball is damaged, the corresponding clearance is:

$$
e_{b}=e+h \text {. }
$$




\section{Results and discussion}

\subsection{Health signal simulation}

When the rolling bearing operates under a radial load, the load distribution changes periodically. When the rolling element is in the asymmetric position under the load, the shaft center not only moves along the vertical direction but also moves along the horizontal direction. Such variations and nonlinear characteristics of the restoring force produce multiple harmonics and their combined vibrations, which are excited by the bearing body. This is called the varying compliance (VC) vibration (HARSHA S P, 2006). In the present study, 6203 deep groove ball bearing is considered as an example. In the experiment, the shaft speed, sampling frequency and VC frequency are set to 1797 $\mathrm{rpm}, 12000 \mathrm{~Hz}$ and $107.7 \mathrm{~Hz}$, respectively. The frequency spectrum in Fig. 4 shows the frequency $\mathrm{fr}=29.95 \mathrm{~Hz}, \mathrm{VC}$ frequency and its harmonics $2 \mathrm{VC}$, 3 VC. It is observed that the analysis model can better simulate the vibration performance of health signals, and the rest of the frequency components are generated by combined vibration.
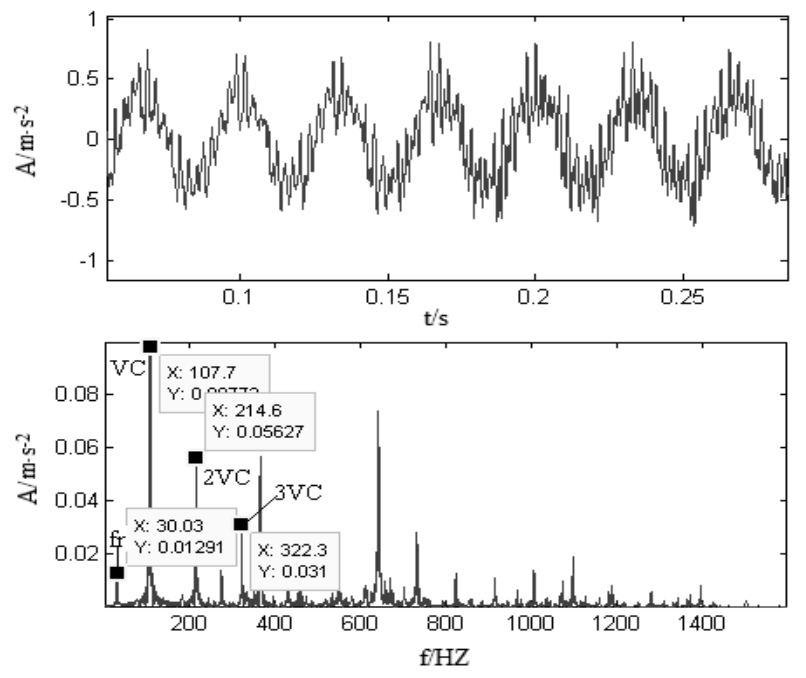

Fig. 4 Vibration response of a bealthy bearing $[8,9]$

\subsection{Damage signal simulation}

With the continuous growth of the bearing service life, the bearing damage gradually deteriorates or degenerates, which can be said that the damage is both a state and a process. In order to simulate the damage evolution process, the damage depth h was set to 0.02 $\mathrm{mm}$, while different widths 1 , including $0.01 \mathrm{~mm}, 0.05$ $\mathrm{mm}$ and $0.09 \mathrm{~mm}$ are considered. Figures 5 to 7 show the simulation signals of the outer ring, inner ring and composite damage, respectively, indicating different degrees of the damage propagation. It is found that as the local damage occurs, the shock attenuation waveform appears in the time domain. The impact period is 1 / fo and $1 / \mathrm{fi}$, which is mainly caused by the instantaneous change of the clearance.
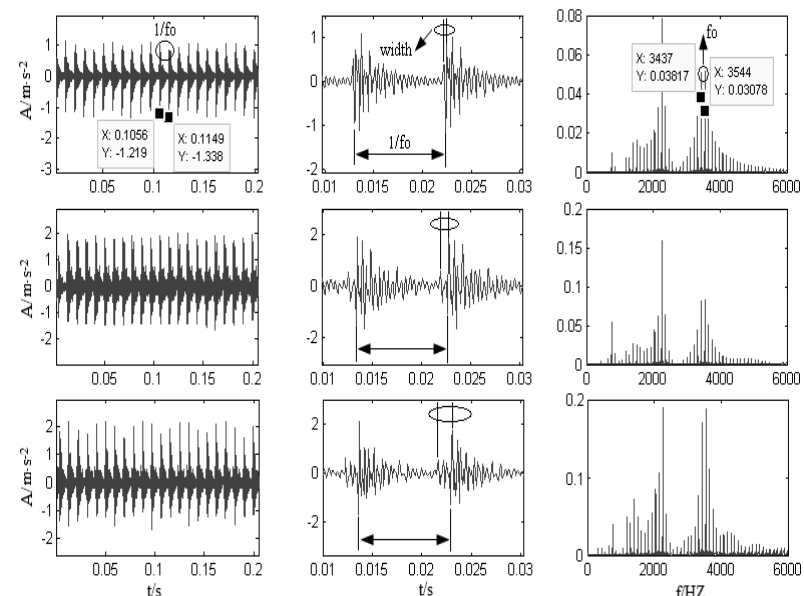

Fig. 5 Vibration response with a defected outer race
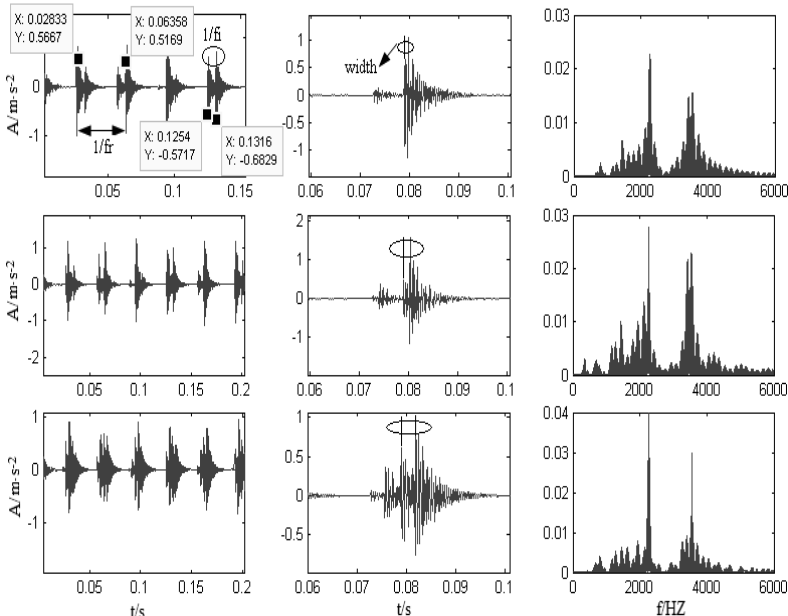

Fig. 6 Vibration response with a defected inner race
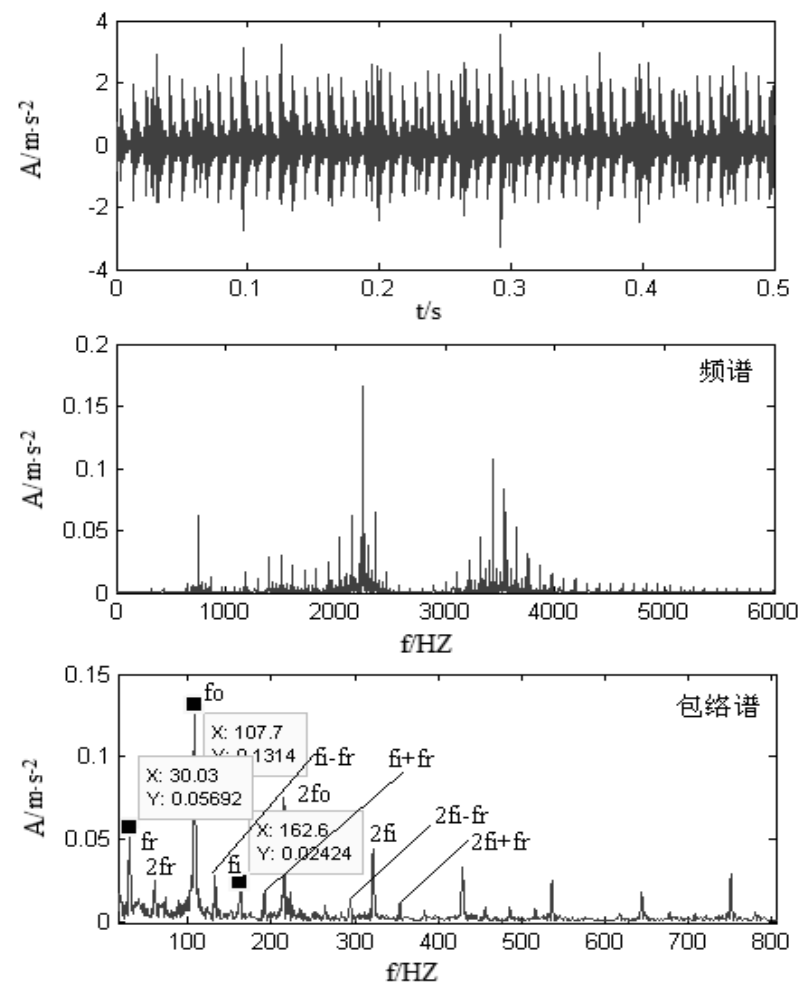

Fig. 7 Vibration response with defected inner and outer races 
Studies show that the bearing surface damage is essentially a contact problem with geometric defects. As the damage intensifies, the damaged area becomes larger and the natural frequency of the elastic contact system decreases. This is equivalent to install a flexible part to change the vibration response. With the increase of the damage severity, the signal amplitude increases, the local attenuation width of the two adjacent shock peaks increases and the energy structure of the spectrum changes. In comparison, the damage vibration of the inner ring is more complex than xxx, because the damage region rotates with the rotating shaft, and the frequency fr modulates the damage frequency and its harmonics.

The dynamic signal of the bearing composite damage is the combination and modulation of two damage characteristics. Since the envelope spectrum can effectively extract the modulation components, the damage frequency fo $=107.7 \mathrm{~Hz}, \mathrm{fi}=162.6 \mathrm{~Hz}$ and the combined frequencies of fi-fr、fitfr、2fo、 $2 \mathrm{fi}$ 、 $2 \mathrm{fi}+\mathrm{fr} 、 2 \mathrm{fi}-\mathrm{fr}$ are presented in the spectrum.

In the present study, three damage areas with a depth of $0.02 \mathrm{~mm}$ and a width of $0.01 \mathrm{~mm}$ were set up. Fig. 8 presents the vibration response in this regard. In indicates that the existence of multiple damages further increases the local flexibility and increases the vibration amplitude. The vibration signal produces a series of characteristic waveforms with the same damage frequency but different phases, which are shown as the damage characteristic frequency in the spectrum and envelope spectrum. Based on the performed analyses, it is found that the embedded integrated modeling is capable of simulating the impact information and damage propagation process of bearing damage effectively and accurately. Moreover, it covers the middle and high-frequency components of the spectrum, which is consistent with the actual situation.
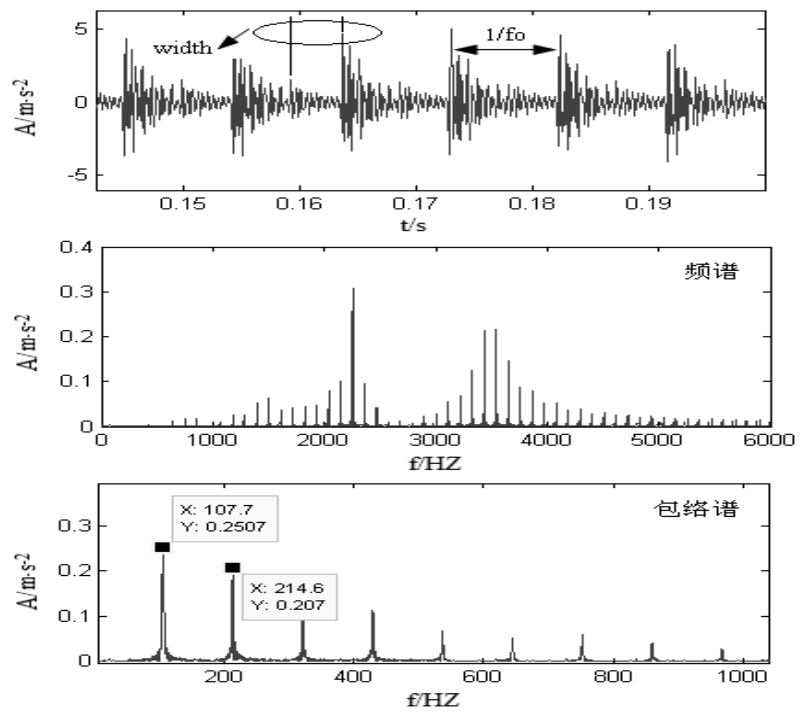

Fig. $\boldsymbol{8}$ Vibration response with a multi-defected outer race

\section{Damage degree assessment}

\subsection{AR-EVA feature enhancement algorithm}

The AR model is a predictor and a filter, which can eliminate fixed periodic components in the bearing signal (Min, 1998). Because of the complexity of the vibration transmission, weak shock characteristics are often submerged by a lot of noise and channel structure. However, applying the blind deconvolution algorithm can identify weak shocks when the transmission characteristics are unknown.

When the blind deconvolution algorithm is applied to $\boldsymbol{e}[n]$, an approximate estimation of the original signal obtained by a finite impulse response filter can be obtained. This can be mathematically expressed as the following:

$$
\begin{gathered}
\tilde{\boldsymbol{x}}[n]=\boldsymbol{e}[n] * \boldsymbol{y}[n] . \\
\tilde{\boldsymbol{x}}[n]=\sum_{m=0}^{l} \boldsymbol{e}[m] \boldsymbol{y}[n-m] .
\end{gathered}
$$

Equations (19) and (20) can be solved through the least square method (LSE) in the form below:

$$
L \mathrm{SE}\left(\mathbf{e}, k_{0}\right)=\mathrm{E}\left\{\left|\tilde{\boldsymbol{x}}(n)-\boldsymbol{x}\left(n-k_{0}\right)\right|^{2}\right\} .
$$

Where $k_{0}$ denotes the delay of the input signal. In order to obtain the filter length, the eigenvector algorithm (EVA) is applied in the present study to solve equation (20) [11]. It should be indicated that the EVA algorithm uses the kurtosis maximization criterion to optimize the objective function so that the filter length can be obtained iteratively. It is assumed that $\boldsymbol{x}_{\mathrm{d}}, \boldsymbol{x}_{\mathrm{f}}$ and $\boldsymbol{x}_{\mathrm{n}}$ stand for fixed component, impact component and random noise, respectively. With these definitions, the block diagram of the feature enhancement based on the AR-EVA algorithm can be expressed as follows:

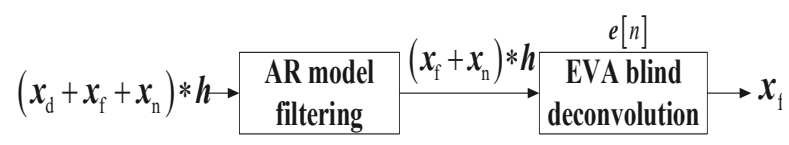

Fig. 9 Block diagram of the AR-EVA algorithm

Figure 10 shows the early damage signal of the rolling element and results of the blind deconvolution process, while the characteristic frequency is $140 \mathrm{~Hz}$. Moreover, Fig. 10(b) indicates that the submerged component of the weak shock in background noise is revived after applying the deconvolution process, while the impact interval is $0.0071 \mathrm{~s}$, which is consistent with the characteristic frequency. 


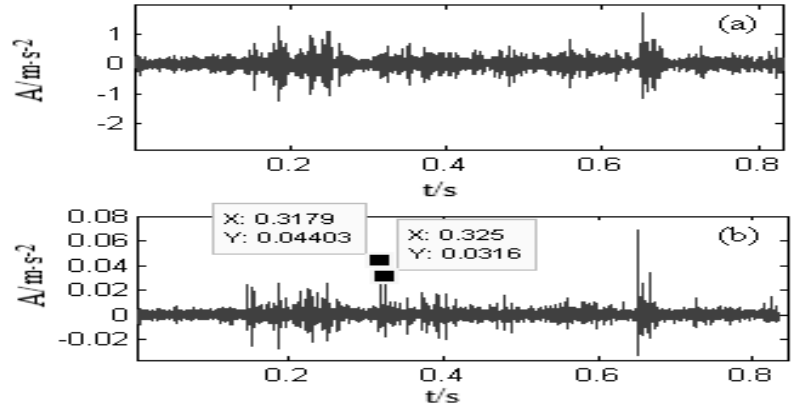

Fig. 10 Comparing the influence of $A R-E V A$ algorithm on the signal: (a) Original signal (b) signal after implementation of the AR-EVA algorithm

\subsection{Solution of the inverse problem}

Based on the AR-EVA feature enhancement method, a standard mode is established by using the timedomain pulse and margin index combined with four dimensionless indices, including the average frequency, spectrum skewness, spectrum kurtosis and root mean square ratio, in the frequency domain (Peng, 2005). Consequently, an inverse problem of the damage assessment is transformed into the optimization problem of minimizing the differences between the actual vibration output of the bearing and the numerical output of the contact vibration model. In this section, the distance function (Min, 1998). was used to find the sample point nearest to the input value. Then the damage location and damage width were measured. Through the positive problem analysis, the following standard database is determined:

$$
\boldsymbol{s}_{i}(k)=\left\{x_{i}(1), x_{i}(2), \cdots x_{i}(6)\right\} \text {. }
$$

Where $i$ is the pattern category. In order to quantitatively detect the damage degree, the concept of the damage interval is proposed to represent the specific damage degree as shown in FIG. 11. In the proposed method, $\mathrm{L}$ value of each interval is divided into $l_{1}, l_{2}$, $l_{3}$ with a certain step size and then the specific dimensions of $l_{1}, l_{2}, l_{3}$ can be calculated. The interval code corresponding to the size is the quantitative evaluation result.

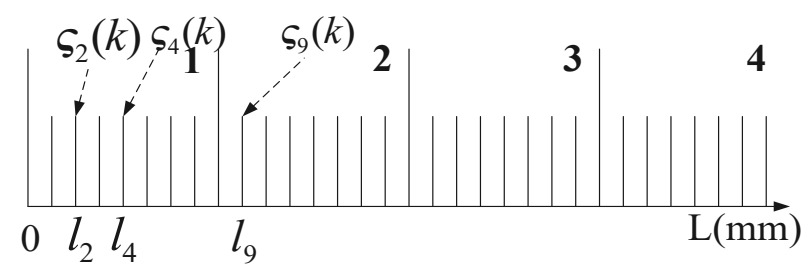

Fig. 11 defects range

The solution of the inverse problem is to determine the specific damage interval. The identification process is described as the following:

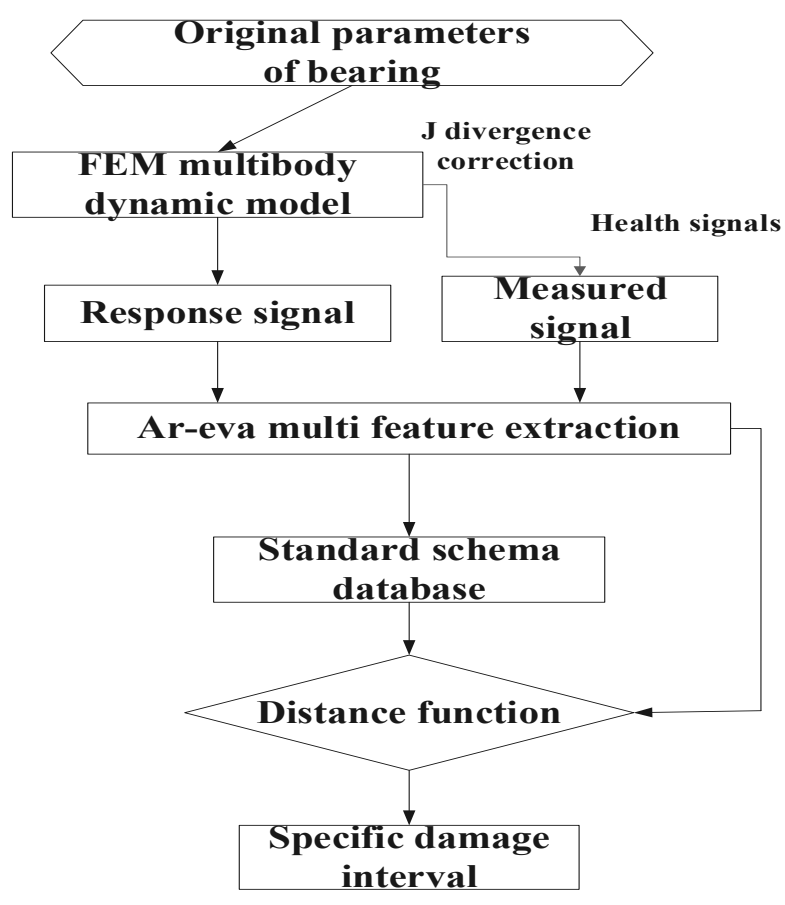

Fig. 12 The flowchart of the quantitative evaluation for rolling bearing

\subsection{Case verification}

In order to verify the calculations, a bearing (Case Western Reserve University (CWRU)) (The Case Western Reserve University Bearing Data Center Website, 2020).is considered as the research objective. In this test, the EDM was used to machine the blind holes with a depth of $11 \mathrm{mils}(0.2794 \mathrm{~mm})$ on the inner, outer ring and the rolling element of the tested bearing. Three types of bearings with blind hole diameters of 7 mils $(0.1778 \mathrm{~mm}), 14 \mathrm{mils}(0.3556 \mathrm{~mm})$ and $21 \mathrm{mils}$ $(0.5334 \mathrm{~mm})$ are utilized in the experiment. The bearings were operated under four different loads within the range of $0-3 \mathrm{hp}$ and rotating speeds of $1797 \mathrm{rpm}$, $1772 \mathrm{rpm}, 1750 \mathrm{rpm}$ and $1730 \mathrm{rpm}$. The vibration signal is collected by a 16 channel data recorder with a sampling frequency of $12 \mathrm{kHz}$. When building the damage database, the positive problem analysis interval is set as $(0-0.1) \mathrm{mm},(0.1-0.2) \mathrm{mm},(0.2-0.4) \mathrm{mm},(0.4-$ $0.5) \mathrm{mm},(0.5-0.6) \mathrm{mm},(0.6-0.7) \mathrm{mm}$, and (0.7-2) $\mathrm{mm}$. The damage degree increases in turn, and the searching step is $0.001 \mathrm{~mm}$. The main objective of the quantitative evaluation is to determine each bearing damage belongs to which interval. It should be indicated that CWRU does not provide rolling element data under the fourth working condition. In the present article, 20 groups of inner, outer ring and rolling element samples under the first three working conditions are used to perform the damage assessment according to the flowchart presented in Fig. 12. Table 1 shows the minimum accuracy of damage location assessment under three working conditions. Moreover, table 2 shows the analysis results under three working conditions. 
Tab. 1 Evaluation result of defects location

\begin{tabular}{c|c|c}
\hline $\begin{array}{c}\text { Inner ring da- } \\
\text { mage }\end{array}$ & $\begin{array}{c}\text { Outer ring da- } \\
\text { mage }\end{array}$ & Ball damage \\
\hline $99.3 \%$ & $98.6 \%$ & $98.2 \%$ \\
\hline
\end{tabular}

Tab. 2 Result of quantitative evaluation

\begin{tabular}{c|c|c}
\hline $\begin{array}{c}\text { Inner ring da- } \\
\text { mage }\end{array}$ & $\begin{array}{c}\text { Outer ring da- } \\
\text { mage }\end{array}$ & $\begin{array}{c}\text { Ball da- } \\
\text { mage }\end{array}$ \\
\hline 0.181 & 0.189 & 0.184 \\
\hline 0.361 & 0.368 & 0.365 \\
\hline 0.539 & 0.543 & 0.546 \\
\hline
\end{tabular}

Obtained results in the foregoing sections show that the prediction error of the damage location is less than $2 \%$, and the damage degree belongs to the range of 2,3,5. After repeated tests, the prediction error is less than $8 \%$. It is concluded that the proposed method can be effectively applied in different case studies to obtain extended damage modes.

\section{Conclusion}

The following conclusions are drawn from the present study:

(1) The vibration response of the rolling bearing is the comprehensive embodiment of the internal geometric correlation and motion correlation under external load. In the present study, a dynamic contact vibration model of the rolling bearing is established, which combines the interface complex constraints and transfer path. The model parameters are modified by $\mathrm{J}$-divergence, and the vibration signals of different damage states are obtained. The model can capture the impact information of the vibration signal when the bearing is damaged and covers the middle and highfrequency components of the spectrum.

(2) The appearance of surface damage makes the clearance change instantaneously, which leads to the introduction of part of contact flexibility and the decrease of the natural frequency of the contact system, which affects the vibration characteristics of bearing. Therefore, different damage locations and degrees will form different damage modes. On the contrary, the damage location and specific damage interval can be obtained by using the change of the mode vector.

(3) The inverse problem in the quantitative diagnosis is transformed into the comparison of geometric distances. The measured eigenvectors are used as the input and the distance function is used to quickly identify the damage interval. The case analysis shows that the maximum recognition error is less than $8 \%$.

(4) When the bearing is damaged, the vibration signal obtained by monitoring is collected. Moreover, several dimensionless indices in time and frequency domain are accurately extracted by using the AR-EVA method. The existence of the damage can be predicted in the early stage of damage. Therefore, hidden dangers can be eliminated as soon as possible, and the service life of the bearing can be optimized to maximize the benefits.

\section{Acknowledgement}

We gratefully acknowledge the valuable cooperation of Dr. Qian Jia and the members of her laboratory in preparing this Application note.

\section{References}

[1] SUNNERSJO C S (1978) Varying compliance vibrations of rolling bearings. Journal of Sound and Vibration 58(3):363-373.

[2] RAFSANJANI A, ABBASION S, FARSHIDIANFAR A (2009) Nonlinear dynamic modeling of surface defects in rolling element bearing systems. Journal of Sound and Viration 319(3-5):1150-1174.

[3] LIEW A, FENG N, HAHN E (2002) Transient rotor dynamic modelling of rolling element bearing systems. Transactions of the ASME, Journal of Engineering for Gas Turbines and Power 124 (4) :984-991.

[4] SOPANEN J, MIKKOLA A (2003) Dynamic model of a deep-groove ball bearing including localized and distributed defects. Part 1: theory, Proceedings of the Institution of Mechanical Engineers Part K: Journal of Multi-body Dynamics (217):201-211.

[5] SOPANEN J, MIKKOLA A (2003) Dynamic model of a deep-groove ball bearing including localized and distributed defects. Part 2: implementation and results, Proceedings of the Institution of Mechanical Engineers Part K: Journal of Multi-body Dynamics (217): 213-223.

[6] HARRIS T A (2001) Rolling bearing analysis. 4th ed. New York: John Wiley and Sons.

[7] QU Liang-sheng (2009) Theory and Methodology for Mechanical Condition Monitoring and Fault Diagnosis. Xi'an:Xi'an JiaoTong University Press.

[8] HARSHA S P (2006) Nonlinear dynamic analysis of a high-speed rotor supported by rolling element bearings. Journal of Sound and Vibration 290(1-2):65-100.

[9] MEVEL B, GUYADER J L (2008) Experiments on routes to chaos in ball bearings. Journal of Sound and Vibration 318(3):549-564.

[10] XU Min, YU He-ji, ZHANG Rui-lin (1998) Handbook of macbinery fault diagnosis. Xi'an:Xi'an JiaoTong University Press. 
[11] JELONNEK B, BOSS D, KAMMEYER K D (1997) Generalized Eigenvector Algorithm for Blind Equalization. Signal Processing 61(3): $237-$ 264.

[12] CHEN Peng, MASATOSHI T, TOSHIO T (2005) Fault diagnosis method for machinery in unsteady operating condition by instantaneous power spectrum and genetic programming. Mechanical Systems and Signal Processing 19(1):175194.

[13] The Case Western Reserve University Bearing Data Center Website. Bearing data center seeded fault test data [EB/OL]. Http: http:/ / www.eecs/cwru/edu/laboratory/ bearing. 